\title{
RESEARCH ON WSRF-BASED RESOURCE MANAGEMENT MODEL AND ENCAPSULATION IN MANUFACTURING GRID
}

\author{
Yu'an $\mathrm{He}^{1,2}$, Tao $\mathrm{Yu}^{1}$, Bin Shen ${ }^{1}$ \\ 1.Cims \& robot Center, Shanghai University, China 200072 2.Shanghai Institute of \\ Technology, China 200233 Email: hya@graduate.shu.edu.cn
}

Abstract: In order to realize resource sharing and collaboration effectively among the heterogeneous and geographical distributed manufacturing resources, a Concept Model of resource management, a WSRF (Web Service Resource Framework)-based Resource Management System Model in Manufacturing Grid (MG) and the resource encapsulation method were presented in this paper. By the two models, the description, encapsulation and publication of manufacturing resources are implemented conveniently, and the manufacturing resource can be encapsulated to a WS (Web Service)-Resource structural MGResource. The encapsulation process shields complexity and diversity of manufacturing resources and implements manufacturing resource sharing and collaboration in MG effectively.

Key words: WSRF; Manufacturing Grid (MG); Resource management; Web service; Resource encapsulation.

\section{INTRODUCTION}

Manufacturing Grid (MG) ${ }^{1}$ is the application of Grid Technology in manufacturing, the core idea of $M G$ is manufacturing resource sharing and collaboration. Manufacturing resources mean the equipments, materials, personnel and all the hardware and software related to the product lifecycle ${ }^{2}$. Manufacturing resources have the characteristics of complexity, diversity and distribution, and manufacturing tasks have many characteristics which are different from those in computing grid. Thus there are many differences between MG and computing grid in resource management mode, the

This paper is Supported by "SEC E-Institute: Shanghai High Institutions Grid" project \& "SEC project: No. 03HK07.

Please use the following format when citing this chapter:

He, Yu'an, Yu, Tao, Shen, Bin, 2006, in International Federation for Information

Processing (IFIP), Volume 207, Knowledge Enterprise: Intelligent Strategies In Product Design, Manufacturing, and Management, eds. K. Wang, Kovacs G., Wozny M., Fang M., (Boston: Springer), pp. 908-913. 
resource management method in $\mathrm{MG}$ is more complicated than that in computing grid.

An effective Manufacturing Grid Resource Management System (MGRMS) is crucial. Document 3 has ever proposed a MG-RMS which can solve the resource sharing and collaboration well under the heterogeneous and distributed environment. But when MG deals with complex manufacturing tasks, resource consumers put forward more requirements for manufacturing service level and manufacturing resources, the MG-RMS can not support sufficiently. So a WSRF (Web Service Resource Framework) ${ }^{4}$-based Resource Management System is proposed in this paper. WSRF is the open criterion of the next generation of grid technology, and it defines the sharing mechanism and management mechanism of grid software and hardware resources.

\section{WSRF-BASE MG-RMS}

\subsection{Concept Model}

Concept Model is the abstract of design, i.e., it is the visualization representation of design idea. According to function roles, there are three main roles related to resource management in MG: resource providers, resource consumers and Manufacturing Grid Information System (MGIS). The relations between the three roles in concept model are shown in Fig.1. Their definitions are as follows:

Resource providers: they are the principal bodies controlling manufacturing resources, and provide some resources to outside in the service manner.

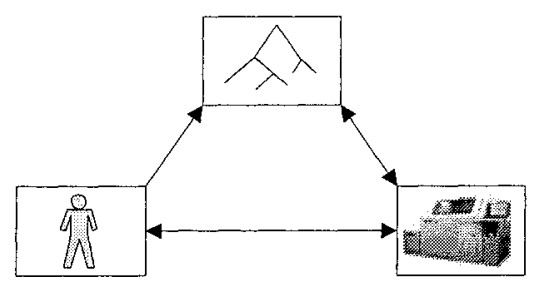

Figure 1. Concept model of MG resource management

MGIS: MGIS is responsible for receiving, sending, indexing and 
archiving the attributes related to MG-Resources, and dealing with the state information.

Resource consumers: they are the users, usually the members of Visual Organization (VO) or customers using resources.

In MG environment, MG-Resource is the model of manufacturing resources encapsulated in MG. In order to realize resource sharing and collaboration among the heterogeneous and geographical distributed manufacturing resources, manufacturing resource must be encapsulated with uniform criterion, and deployed to MGIS.

\subsection{System Model}

There are varieties kinds of manufacturing resources, which have great differences among them. In order to solve the geographical distributed sharing problem of manufacturing resources, MG-RMS is developed.

The system model of MG-RMS is shown in Fig.2. It is mainly composed of WSRF encapsulation module, MG-Resource, Manufacturing Grid Information System (MGIS) and task manager in a resource node, and its application module includes security management, task decomposing, Manufacturing Grid Resource Scheduler (MGRS), workflow manager, MG portal and MG-QoS (Quality of Service)manager.

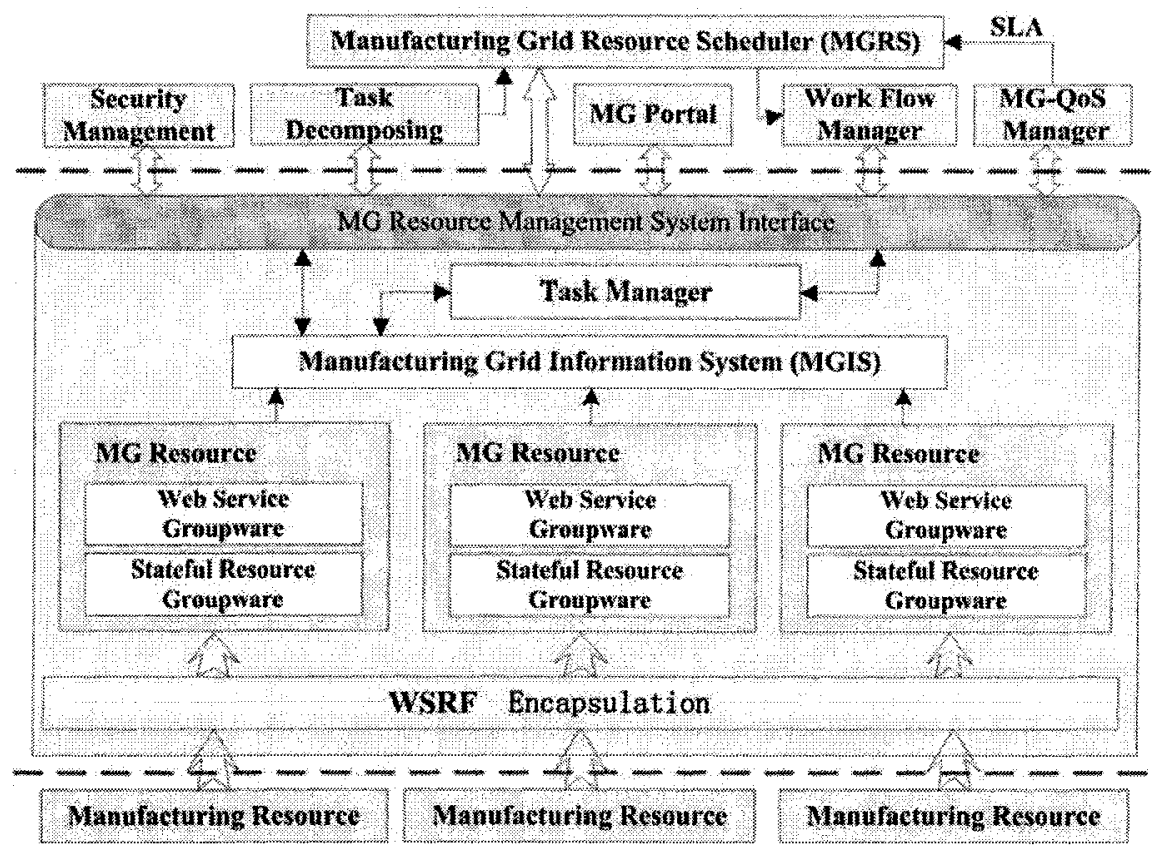

Figure 2. The system model of MG-RMS 
1) WSRF encapsulation module: According to the requirements of MGRMS, WSRF encapsulation module uses WSRF criterion to encapsulate manufacturing resources into MG-Resources, publishes them to the MG platform so as to be invoked by consumers who need these resources.

2) MG-Resource: MG-Resource is the mapping and representation of actual manufacturing resource in MG. All the resources which hope to share in MG need to be encapsulated into MG-Resources and published on the MG platform. Manufacturing resources can be all the software and hardware related to the product lifecycle, e.g., a machine tool, a set of CAD software, even an expert or machining personnel.

3) MGIS: MGRIS transfers attribute information of manufacturing resources in MG. It collects the attribute information (including dynamic and static attribute) then publishes them to consumers according to the requirements, so as to choose a proper manufacturing resource to fulfill the manufacturing task. There are several modes to publish information, and resource providers can choose the mode which they need.

4) MG-RMS interface: the application modules, e.g., security management, MGRS, workflow manager and QoS manager, exchange information through the interface. The interface is by means of Web service to exchange information with these applications.

5) Task manager: Task manger receives the task from work flow manager, chooses manufacturing resources to work in MG-RMS according to task's description, and submits to work flow manager after the task is accomplished.

6) Application modules

MGRS: its functions are mainly composed of QoS-based resource and scheduling, dynamic negotiation, resource allocation and fault-tolerant handling.

MG portal: it publishes MG-Resource attribute information to consumers by HTML (HyperText Markup Language) pages.

Workflow module: it invokes a certain MG-Resource and implements the manufacturing task in appropriate time, and submits the task to workflow after the task is accomplished.

Security module: it monitors the reliability and dynamic attribute of MGResource by invoking web service groupware.

Task decomposing module: it is in charge of decomposing a complicated task into many subtasks, so as to search and match resources.

MG-QoS manager: it is the management module of MG Quality of Service (QoS), and its functions include management of Service Level Agreement (SLA), resource reservation and management of QoS strategy. 
The WSRF encapsulation module is the crucial module, so the paper mainly presents the encapsulation method.

\section{RESOURCE-ENCAPSULATION MOTHOD}

The WSRF encapsulation module in MG-RMS encapsulates the actual manufacturing resources into MG-Resources by using WSRF criterion.

MG-Resource is a kind of WS-Resource-structure resource, it includes two modules: web service and stateful resource. Web service can realize the function of MG-Resource, provide uniform and standard external invocation interfaces, and make customers invoke MG-Resources by sending standard web service. The stateful resources can save the attribute information about manufacturing resources, publish the information to customers, and make them discover manufacturing resources which are suitable for machining the manufacturing task ${ }^{6}$.

Thus, encapsulating manufacturing resources to MG-Resources includes two parts: web service encapsulation and statful resource encapsulation. A WSRF-based MG resource encapsulation structure is shown in Fig.3.

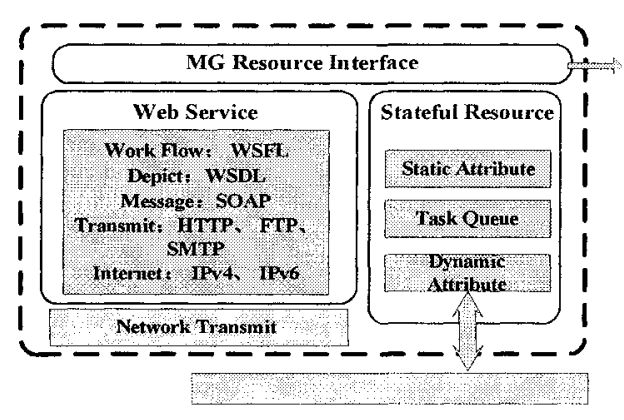

Figure 3. The resource encapsulation model

\subsection{The Encapsulation of Stateful Resource}

According to the characteristics of attribute information, manufacturing resource information can be divided into three types of information, that is, static attribute information, dynamic attribute information and task queue information, which are crucial for the resource evaluation and scheduling.

Since the quantities of manufacturing resources published in a local MGRMS are limited, the stateful resources are implemented to records in the Microsoft Access database. 


\subsection{The Encapsulation of Web Service}

The web service module of MG-Resource takes charge of the realization of the functions of manufacturing resources, and management of attribute information of manufacturing resources. Web service is a group of objects deployed on web, provides standard external interface format by WSDL (Web Service Description Language) document. Applicants may send standard SOAP (Simple Object Access Protocol) message to web service so as to invoke these methods.

The encapsulation of web service may carry out according to following steps: 1) Defining interfaces of web service. 2) Creating WSDL document according to the defining interface. 3) Realization of interface document. 4) Compiling the deploying document and publishing the web service on the Axis Server.

\section{CONCLUSION}

As has been noted, the WSRF-based MG-RMS model constructed by using WSRF criterion can describe, encapsulate and publish manufacturing resources, and the resource is encapsulated to a WS (Web Service)-Resource structural MG-Resource. The MG-RMS can solve resource sharing problem among the heterogeneous and geographical distributed manufacturing resources, make consumers invoke the resources through the standard interface conveniently, as if the manufacturing resources are in local place.

\section{REFERENCES}

1. Liu Lilan, Yu Tao, Shi Zhanbei. Research of Self-Organization Manufacturing Grid and its Task Scheduling Algorithm, Computer integrated manufacturing system (CIMS), 2003, 9 (6): 449-455

2. Zhou Guanghui, Jiang Pingyu. The Encapsulation and Integration of Networked Manufacturing Resource Based on Mobile Agent. Computer Integrated Manufacturing System (CIMS). 2002, 9(8): pp 530-538

3. LIU Lilan, YU Tao, SHI Zhan. Research on QoS-based Resource Scheduling in Manufacturing Grid, Computer Integrated Manufacturing System (CIMS). 2005, 11(4): pp 475-480

4. The WS-Resource Framework. Available: http://www.globus.org/wsrf/specs/ws-wsrf.pdf

5. He Yu'an, Yu Tao, Liu Lilan, et al. A QoS-based Task Decomposing Middleware Research for Manufacturing Grid. Proceedings of the First International Conference on Semantics, Knowledge and Grid(SKG05), Beijing, China, 2005, 513-519

6. He Yu'an, Yu Tao, Liu Lilan, et al. A WSRF-based Resource Management System of Manufacturing Grid. Proceedings of 6th IEEE International Symposium on Cluster Computing and the Grid (CCGrid(06), Singapore, 2006 